\title{
LA TRANSFERENCIA DE LA EVALUACIÓN FORMATIVA Y/O COMPARTIDA DESDE LA FORMACIÓN INICIAL DEL PROFESORADO DE EDUCACIÓN FÍSICA A LA PRÁCTICA REAL EN EDUCACIÓN PRIMARIA
}

The transfer of the formative and shared assessment from pre-service physical education teacher education to real practice in primary education

Transferência da avaliação formação e compartilha do formação inicial de professor do educação física verdadeira prática no ensino primário

Proyecto de I+D+i: "La competencias docentes en la formación inicial del profesorado de educación física”. Convocatoria de noviembre de 2013 del Programa Estatal de Investigación, Desarrollo e Innovación Orientada a los Retos de la Sociedad, en el marco del Plan Estatal de Investigación Científica y Técnica y de Innovación 2013-2016. Referencia: EDU 2013-42024-R. Duración: 3 años (2014-2016).

\section{Miriam Molina Soria (1)}

\section{Víctor Manuel López Pastor (2)}

(1) Universidad de Valladolid, España. Teléfono: +34 650631080. Correo electrónico: molina_valsain@hotmail.com

(2) Universidad de Valladolid, España. Correo electrónico: vlopez@mpc.uva.es

\begin{abstract}
Resumen
Esta investigación tiene como finalidad analizar la transferencia entre los sistemas de evaluación formativa y/o compartida que el profesorado de educación física (EF) puede haber vivido durante su formación inicial (FIP) y su aplicación posterior en las aulas de educación primaria en que ejerce su docencia actualmente. Se ha aplicado una metodología mixta, con una muestra reducida. Las técnicas de obtención de datos son: cuestionario cerrado con escala tipo Likert, entrevistas semi-estructuradas y un grupo focal. Los resultados parecen indicar que sí existe cierta transferencia entre los sistemas de evaluación vividos en FIP y los que posteriormente se utilizan en la práctica posterior en educación primaria. También parece existir una influencia fuerte de la formación permanente.

La transferencia de la evaluación formativa y compartida desde la formación inicial del profesorado de educación física a la práctica real en Educación Primaria
\end{abstract}


Palabras claves: Evaluación formativa; evaluación compartida; formación del profesorado; educación física

\begin{abstract}
The purpose of this research is to analyze the transference between the formative and shared assessment that Physical Education (PE) teachers may have experienced during their Pre-service Teacher Education (PTE) and its subsequent application in primary education classrooms where they are teachers. A mixed methodology has been used, with a reduced sample. The data collection techniques are: closed questionnaire with Likert-type scale, semi-structured interviews and a focus group. The results seem to indicate that there is some transference between the assessment systems lived in PTE and those that are later used later when they are PE teachers in Primary. There also seems to be a strong influence of In-service Teacher Education.
\end{abstract}

Keywords: Formative Assessment; Shared Assessment; Teacher Education; Physical Education

\title{
Resumo
}

Esta pesquisa é analisar a transferência entre os sistemas de avaliação formativa e / ou que compartilharam professores de educação física (PE) pode ter vivido durante a sua formação inicial (IFJ) e sua posterior aplicação em salas de aula do ensino fundamental em exercer seu ensinamento hoje. Tem sido aplicada uma metodologia misto, com uma amostra reduzida. As técnicas de recolha de dados são fechadas escala de Likert, entrevista semi-estruturada e um questionário grupo foco. Os resultados sugerem que existe realmente alguma transferência entre os sistemas de avaliação viveu no FIP e, posteriormente, utilizados na prática subsequente no ensino primário. Também parece ser uma forte influência da formação de professores.

Palavras-chave: avaliação formativa; avaliação compartilhada; formação de professores; educação física

\section{Introducción}

Existen pocos estudios sobre la posible transferencia entre experimentar sistemas de evaluación formativa y compartida (EFyC) durante la FIP y su posterior aplicación en la 
futura práctica profesional (Hamodí \& López-Pastor, 2012; Hamodí, López-Pastor \& López-Pastor, 2017; Palacios \& López-Pastor, 2013). Todos ellos nos indican que sí existe cierta trasferencia, pero que también aparecen sensaciones de inseguridad del profesorado los primeros cursos que lo aplican en educación primaria.

Por otra parte, algunos estudios parecen indicar que al profesorado en formación le pesa mucho más su larga experiencia como estudiante que la formación específica recibida sobre el tema. Es decir, tienden a reproducir lo que han hecho con ellos o ellas cuando han sido alumnos (en este caso, la obsesión por la calificación, por la nota, o la preeminencia de una evaluación exclusiva o principalmente sumativa y calificativa, en vez de formativa), en lugar de aplicar lo aprendido durante su FIP (Palacios \& LópezPastor, 2013).

López-Pastor (2012) define algunos de los conceptos básicos sobre EFyC en la FIP: evaluación para el aprendizaje; mientras que Martínez-Mínguez, Vallés y RomeroMartín (2015) detallan las ventajas que parece tener el desarrollo sistemas de EFyC.

Esta investigación busca estudiar los posibles procesos de trasferencia entre los sistemas de EFyC que el profesorado de EF puede haber vivido durante su FIP y su aplicación posterior en las aulas de educación primaria en que ejerce actualmente. Por tanto, los objetivos de investigación son los siguientes:

1-Analizar si el profesorado de EF de primaria utiliza sistemas de EFyC en sus aulas.

2-Analizar en qué medida las experiencias de evaluación vividas durante la FIP de los maestros de EF ha influido en el tipo de evaluación que realizan actualmente en sus aulas.

\section{Método}

Participan 18 maestros de EF de la E.U. de Magisterio de Segovia. La metodología es mixta, combinando las siguientes técnicas de obtención de datos: cuestionario estructurado inicial, entrevista semiestructurada y grupo focal.

El cuestionario consta de 45 ítems que preguntan sobre los sistemas de evaluación utilizados habitualmente, así como por la participación del alumnado. Utiliza 
una escala Likert, en la que: 1=nada de acuerdo; 2=poco de acuerdo; 3=bastante de acuerdo; 4=muy de acuerdo.

El análisis de los datos cuantitativos lo realizaremos con el programa SPSS.20.0, mientras que para los datos cualitativos utilizaremos el atlas.ti 8.0.

En estos momentos solo disponemos de los datos de algunos cuestionarios iniciales y el grupo focal. En el apartado de resultados presentamos estos datos y en el congreso presentaremos la totalidad de ellos. Las entrevistas semiestructuradas se llevarán a cabo entre los meses de abril y mayo, por lo que aportaremos estos resultados completos en el congreso.

\section{Resultados y discusión}

Hasta el momento, los cuestionarios recopilados de los maestros de EF que realizan EFyC coinciden en la importancia de ésta en el proceso de enseñanza aprendizaje y, en muchos casos, en la necesidad de la participación del alumnado en el proceso de evaluación y de calificación. Más o menos todos afirman realizar procesos de evaluación formativa, bien de forma absoluta (todos los procesos de evaluación llevados a cabo son formativos) o parcial (utilizan a veces procesos de evaluación formativa, pero no siempre), pero en cambio son menos los que llevan a cabo procesos de evaluación compartida, favoreciendo la participación del alumnado en la evaluación.

En los egresados más jóvenes, sí indican que vivieron alguna experiencia de EFyC durante su FIP (en diferentes centros), y que dicha experiencia les ha influido en tender a realizar ese tipo de sistemas de evaluación en su práctica docente. En cambio, hay otro grupo de profesores que no tuvieron este tipo de experiencias durante su FIP, pero también han llegado a desarrollar sistemas de evaluación formativa en su práctica profesional; en general, este último grupo de egresados suele haber terminado su FIP hace ya muchos años.

En la Tabla 1 presentamos los resultados más importantes obtenidos en un grupo focal realizado con egresados recientes de las facultades de educación que han experimentado sistemas de EFyC durante su FIP. Hemos seleccionado dos temas: (a) evaluación en la universidad; (b) evaluación formativa y/o compartida en los centros. 
Tabla 1.

Resultados del grupo focal sobre evaluación formativa y competencias docentes en la formación inicial del profesorado.

Evaluación en la universidad

Se exige evaluar y trabajar por competencias que vienen impuestas previamente. Aunque no se apliquen correctamente, se puede afirmar que los graduados actuales se sienten más competentes que los que terminaron hace años.

La evaluación en la universidad sigue siendo la misma de siempre en la mayoría de las asignaturas: se realiza un examen final valorado con un alto porcentaje de la calificación final y las prácticas con un mínimo porcentaje.

La evaluación formativa y/o compartida solo se lleva a cabo en la universidad por un reducido número de profesores.

\section{Conclusiones}

Nuestros resultados provisionales muestran que sí parece existir una transferencia entre haber vivido sistemas de EFyC durante la FIP y su aplicación posterior en las aulas de educación primaria. La mayoría de los maestros que han participado en el estudio sí utilizan sistemas de evaluación formativa en sus aulas, pero son menos los que también favorecen la participación del alumnado en la evaluación.

Estos resultados parecen indicar la importancia de que durante la FIP se experimenten sistemas de EFyC. La limitación del estudio es que la muestra es muy reducida y local; por tanto sería interesante realizar estudios similares con muestras más grandes, o bien en otros contextos geográficos, con maestros que se hayan formado en otras facultades.

\section{Referencias}

Hamodi, C., \& López-Pastor, A.T. (2012). La evaluación formativa y compartida en la Formación Inicial del Profesorado desde la perspectiva del alumnado y de los egresados. Psychology, Society, \& Education, 4(1), 99-112.

Hamodi, C., López-Pastor, A. T., \& López-Pastor, V. M. (2017). If I experience formative assessment whilst at University will I put it into practice later as a teacher? Formative and shared assessment in Initial Teacher Education (ITE).

La transferencia de la evaluación formativa y compartida desde la formación inicial del profesorado de educación física a la práctica real en Educación Primaria 
European Journal of Teacher Education, 40(2), 171-190. DOI: 10.1080/02619768.2017.1281909

López-Pastor, V. M. (2012). Evaluación formativa y compartida en la universidad: clarificación de conceptos y propuestas de intervención desde la Red Interuniversitaria de Evaluación Formativa. Phsychology, Society \& Education, 4(1), 117-130.

Martínez-Mínguez, L., Vallés-Rapp, C., \& Romero-Martín, M. R. (2015). Estudiantes universitarios: ventajas e inconvenientes de la evaluación formativa. Revista d'Innovació educativa 14, 59-70.

Palacios, A., \& López-Pastor, V. M. (2013) Haz lo que yo digo pero no lo que yo hago: sistemas de evaluación del alumnado en la formación inicial del profesorado. Revista de Educación, 361, 279-305. 